\title{
Local equivalence of transversals in matroids
}

\author{
D. Fon-Der-Flaass * \\ Mathematics Department, \\ Queen Mary and Westfield College, \\ London E1 4NS \\ email: d.g.flaass@qmw.ac.uk
}

Submitted: May 21, 1996; Accepted: August 2, 1996.

\begin{abstract}
Given any system of $n$ subsets in a matroid $M$, a transversal of this system is an $n$-tuple of elements of $M$, one from each set, which is independent. Two transversals differing by exactly one element are adjacent, and two transversals connected by a sequence of adjacencies are locally equivalent, the distance between them being the minimum number of adjacencies in such a sequence.

We give two sufficient conditions for all transversals of a set system to be locally equivalent. Also we propose a conjecture that the distance between any two locally equivalent transversals can be bounded by a function of $n$ only, and provide an example showing that such function, if it exists, must grow at least exponentially.
\end{abstract}

Let $M$ be a matroid, and $\mathcal{V}=\left(V_{1}, \ldots, V_{n}\right)$ a collection of subsets of $M$. By a transversal of $\mathcal{V}$ we mean a sequence $\left(v_{1}, \ldots, v_{n}\right)$ of elements of $M$ such that $v_{i} \in V_{i}$ for $i=1, \ldots, n$, and $v_{1}, \ldots, v_{n}$ are independent. By the well-known Rado's Theorem, transversals exist if and only if the following condition is satisfied:

$$
\text { For every } X \subseteq\{1, \ldots, n\}, \operatorname{rank}\left(\bigcup_{i \in X} V_{i}\right) \geq|X| \text {. }
$$

We say that a transversal $\left(v_{1}^{\prime}, \ldots, v_{n}^{\prime}\right)$ is a (result of a) local replacement of $\left(v_{1}, \ldots, v_{n}\right)$ at $i$ if $v_{j}^{\prime}=v_{j}$ for $j \neq i$; and call two transversals locally equivalent if one can be obtained from the other by a sequence of local replacements; the length of the shortest such sequence being the distance between the transversals.

In this note we address two questions: under what conditions are all transversals of a collection $\mathcal{V}$ locally equivalent; and how big (in terms of $n$ ) can be the distance between two locally equivalent transversals. Also, we shall consider in more detail the case when $M$ is the free matroid (the matroid having no cycles).

${ }^{*}$ On leave from Institute of Mathematics, Novosibirsk, Russia; partially supported by the grant 96-01-01614 of the Russian Foundation for Fundamental Research. 


\section{Sufficient conditions of local equivalence}

Here we shall prove two sufficient conditions for all transversals of a set system to be locally equivalent.

THEOREM 1 If a collection $\mathcal{V}=\left(V_{1}, \ldots, V_{n}\right)$ of subsets of a matroid $M$ is such that

$$
\text { For every } \emptyset \neq X \subseteq\{1, \ldots, n\}, \operatorname{rank}\left(\bigcup_{i \in X} V_{i}\right)>|X|
$$

then all transversals of $\mathcal{V}$ are locally equivalent.

The second theorem is a straightforward generalization of a result proved in [1]. Call a subset $V$ of $M$ thick if for every flat $A$ of $M$ either $V \subseteq A$, or $|V \cap A|<|V| / 2$.

THEOREM 2 If $V_{1}, \ldots, V_{n}$ are thick subsets of $M$ and $\mathcal{V}=\left(V_{1}, \ldots, V_{n}\right)$ satisfies (1) then all transversals of $\mathcal{V}$ are locally equivalent, and the distance between any two of them does not exceed $2 n-1$.

Some examples of thick subsets: one-element sets, cycles of size 3, subspaces of an $n$-dimensional vector space over $\mathrm{GF}(2)$.

A partial case of Theorem 2 for $M$ a linear vector space over the field $G F(2)$, and $V_{i}$ one- or two-dimensional subspaces was proved in [1] and independently in [4]. The proof from [1], almost unchanged, applies to the general situation.

We use the notation $\langle X\rangle$ for $X \subseteq M$ to mean the flat in $M$ generated by $X$.

\section{PROOF OF THEOREM 1.}

Let $\bar{x}=\left(x_{1}, \ldots, x_{n}\right)$ and $\bar{y}=\left(y_{1}, \ldots, y_{n}\right)$ be two transversals of $\mathcal{V}=\left(V_{1}, \ldots, V_{n}\right)$. Let $D=D(\bar{x}, \bar{y})=\left\{i \mid x_{i} \neq y_{i}\right\}$. We shall prove that $\bar{x}$ and $\bar{y}$ are locally equivalent by induction on $d=|D(\bar{x}, \bar{y})|$.

First we introduce some notation:

$I=\{1, \ldots, n\}$

$X_{J}=\left\langle x_{j} \mid j \in J\right\rangle$ where $J \subseteq I$;

for $v \in\langle\bar{x}\rangle$, let $I_{X}(v)$ be the smallest set $J \subseteq I$ such that $v \in X_{J}$. (This set is uniquely determined.)

Suppose first that $\left\langle x_{1}, \ldots, x_{n}\right\rangle \neq\left\langle y_{1}, \ldots, y_{n}\right\rangle$. This means that $y_{i} \notin\left\langle x_{1}, \ldots, x_{n}\right\rangle$ for some $i$. Then the sequence $\bar{x}^{\prime}=\left(x_{1}, \ldots, x_{i-1}, y_{i}, x_{i+1}, \ldots, x_{n}\right)$ is an independent transversal of $\mathcal{V}$. It is a local replacement of $\bar{x}$ at $i$; and $\left|D\left(\bar{x}^{\prime}, \bar{y}\right)\right|<|D(\bar{x}, \bar{y})|$. By induction, we are done in this case.

So, let $X=\left\langle x_{1}, \ldots, x_{n}\right\rangle=\left\langle y_{1}, \ldots, y_{n}\right\rangle$. We construct inductively the sequence $\emptyset=I_{0} \subseteq I_{1} \subseteq \ldots \subseteq I_{r} \subseteq I$ as follows:

$I_{k+1}=I_{k} \cup\left\{j \in I \backslash I_{k} \mid V_{j} \nsubseteq X_{I \backslash I_{k}}\right\}$;

$r$ is the first index for which $I_{r} \cap D \neq \emptyset$.

The property (2) implies that $I_{k} \subset I_{k+1}$ for all $0 \leq k<r$; in particular, the length of the sequence does not exceed $n-d+1$, and the number $r$ is well-defined. 
Now we construct a sequence $\left(i_{1}, \ldots, i_{r}\right)$ of indices, and a sequence $\left(v_{1}, \ldots, v_{r}\right)$ of elements $v_{k} \in V_{i_{k}}$; starting with $i_{r}$ and $v_{r}$. Choose any $i_{r} \in I_{r} \cap D$, and $v_{r} \in V_{i_{r}} \backslash X_{I \backslash I_{r}}$.

The choice of $i_{r}$ and $v_{r}$ implies that $i_{r} \in I_{r} \backslash I_{r-1}$, and that the set $I_{X}\left(v_{r}\right)$ contains an element $j \in I_{r-1}$ for which $V_{j} \nsubseteq X_{I \backslash I_{r-1}}$. Set $i_{r-1}=j$, and choose $v_{r-1} \in V_{j} \backslash X_{I \backslash I_{r-1}}$. Again we have $i_{r-1} \in I_{r-1} \backslash I_{r-2}$, and the set $I_{X}\left(v_{r-1}\right)$ contains an element $j \in I_{r-2}$ for which $V_{j} \nsubseteq X_{I \backslash I_{r-2}}$. We continue in the same manner, and finally find $i_{1} \in I_{1}$ and $v_{1} \in V_{i_{1}}$ such that $v_{1} \notin\langle X\rangle$.

To simplify the notation, let us assume that $i_{1}=1, \ldots, i_{r}=r$.

Now we shall perform certain local replacements of both $\bar{x}$ and $\bar{y}$. Consider the sequences $\bar{x}^{(i)}=\left(v_{1}, \ldots, v_{i}, x_{i+1}, \ldots, x_{n}\right)$ and $\bar{y}^{(i)}=\left(v_{1}, \ldots, v_{i}, y_{i+1}, \ldots, y_{n}\right)$ for $i=1, \ldots, r$. Let also $\bar{x}^{(0)}=\bar{x}, \bar{y}^{(0)}=\bar{y}$.

By the choice of the elements $v_{i}$, we have $v_{i} \notin\left\langle x_{i}, \ldots, x_{n}\right\rangle$, and for $i \geq 2, v_{i} \in$ $\left\langle x_{i-1}, \ldots, x_{n}\right\rangle$. Therefore all the sequences $\bar{x}^{(j)}, 0 \leq j \leq r$, are transversals, and are locally equivalent to $\bar{x}$.

If we have $\left\langle\bar{x}^{(i)}\right\rangle=\left\langle\bar{y}^{(i)}\right\rangle$ for $i=1, \ldots, j$ then all the sequences $\bar{y}^{(i)}, 0 \leq i \leq j$, are also transversals, and are locally equivalent to $\bar{y}$. If this holds for $j=r$ then $\left|D\left(\bar{x}^{(r)}, \bar{y}^{(r)}\right)\right|=|D(\bar{x}, \bar{y})|-1$, and $\bar{x}$ and $\bar{y}$ are locally equivalent by induction. On the other side, if $i$ is the first value for which $\left\langle\bar{x}^{(i)}\right\rangle \neq\left\langle\bar{y}^{(i)}\right\rangle$ then $\bar{x}^{(i)}$ is locally equivalent to $\bar{y}^{(i)}$ by the argument from the beginning of this proof. Thus, in either case $\bar{x}$ is locally equivalent to $\bar{y}$, and the theorem is proved.

\section{PROOF OF THEOREM 2.}

The proof below exactly follows the proof of Proposition 5.1 in [1].

Take any two bases $\bar{x}=\left(x_{1}, \ldots, x_{n}\right)$ and $\bar{y}=\left(y_{1}, \ldots, y_{n}\right)$. Suppose that $x_{i} \neq y_{i}$ for some $i$. Let $X=\left\langle x_{j} \mid j \neq i\right\rangle$, and $Y=\left\langle y_{j} \mid j \neq i\right\rangle$. As $x_{i} \notin X$ and $y_{i} \notin Y$, and using the fact that $V_{i}$ is thick, we have

$$
\left|V_{i} \backslash(X \cup Y)\right| \geq\left|V_{i}\right|-\left|V_{i} \cap X\right|-\left|V_{i} \cap Y\right|>\left|V_{i}\right|-\left|V_{i}\right| / 2-\left|V_{i}\right| / 2=0 .
$$

Therefore there exists an element $z$ of $V_{i}$ which belongs to neither $X$ nor $Y$; and we can replace both $x_{i}$ and $y_{i}$ by $z$. Thus, using at most two local replacements, we can reduce by 1 the number of places in which $\bar{x}$ and $\bar{y}$ disagree.

This argument gives an upper bound of $2 n-1$ on the maximum distance between transversals; because at the last stage, when $\bar{x}$ and $\bar{y}$ differ in only one place, one needs only one local replacement, and not two.

The proof of Theorem 1 also gives an upper bound on the distance between transversals; the distance cannot exceed

$$
2+4+\ldots+2(n-1)+1=n^{2}-n+1 .
$$

By all probability, this bound is far from sharp. It would be very interesting to

find the exact bound on the distance between transversals under the assumptions of Theorem 1. 


\section{$2 \quad$ Free matroid}

A free matroid $M$ is a matroid with no cycles. In a free matroid all subsets are independent; and $\operatorname{rank}(X)=|X|$ for all $X \subseteq M$. Let $\mathcal{V}=\left(V_{1}, \ldots, V_{n}\right)$ be a collection of subsets of $M$ which has at least one transversal. Let $N=\{1, \ldots, n\}$. By the kernel of $\mathcal{V}$ we shall mean the largest subset $X \subseteq N$ for which

$$
\left|\bigcup_{i \in X} V_{i}\right|=|X|
$$

The kernel exists since if $X_{1}$ and $X_{2}$ satisfy this property then so does $X_{1} \cup X_{2}$.

THEOREM 3 Two transversals of $\mathcal{V}$ are locally equivalent if and only if they agree on the kernel $K$ of $\mathcal{V}$; the distance between them then does not exceed $2(n-|K|)-1$.

PROOF. We construct a bipartite graph $G=(N \cup M, E)$ with parts $N$ and $M ; i \in N$ is adjacent to $v \in M$ when $v \in V_{i}$. The transversals of $\mathcal{V}$ are in one-to-one correspondence with the matchings in $G$ covering the part $N$; and a local replacement in this language corresponds to changing a single edge in the matching.

Let $L$ be the set of vertices in $M$ adjacent to vertices from $K$; by definition of $K$ we have $|L|=|K|$. Thus, no edge incident to a vertex in $K$ can ever be changed; and the "only if" part of the theorem is proved.

The set system corresponding to the graph induced on $(N \backslash K) \cup(M \backslash L)$ has an empty kernel, and at least one transversal. Thus, if $K \neq \emptyset$ then we can apply induction on $|N|$. So we assume that $K=\emptyset$, i.e. $\mathcal{V}$ satisfies the conditions of Theorem 1 .

Let $A=\left(a_{i} \mid i \in N\right)$ and $B=\left(b_{i} \mid i \in N\right)$ be any two transversals. Colour the edges $\left(i, a_{i}\right)$ blue, and $\left(i, b_{i}\right)$ red. The multigraph formed by all coloured edges is a disjoint union of cycles (possibly of length 2 if $a_{i}=b_{i}$ for some $i$ ), paths, and isolated vertices. Let $\left\{C_{1}, \ldots, C_{k}\right\}$ be the set of all its cycles. We shall prove by induction on $n+k$ that the distance between $A$ and $B$ is at most $n+k$. Since $k \leq n$, and $k=n$ only if $A=B$, this will prove the theorem.

Let $C=\cup C_{i}, X=C \cap N, Y=C \cap M$. We have $|X|=|Y|$. Applying the inequality 2 to the set $X$ we see that in $G$ there is an edge between $X$ and $M \backslash Y$; colour it green. This edge is incident to exactly one of the cycles $C_{1}, \ldots, C_{k}$; we delete it from $C$ and apply the same argument to the set of remaining cycles; and continue in the same manner until we get $k$ green edges. We shall consider the subgraph $G^{\prime}$ of $G$ formed by all coloured edges (blue, red, and green). The system corresponding to $G^{\prime}$ also satisfies the property 2 , and both $A$ and $B$ are its transversals.

Suppose first that there exists a green edge $p q, p \in N, q \in M$ such that $q$ is not incident to any red edge. Then we can perform one local replacement on $B$ replacing the red edge incident to $p$ by $p q$, and reduce the number of cycles by 1 . By induction, we are done in this case. Similarly we treat the case when $q$ is not incident to any blue edge. 
THE ELECTRONIC JOURNAL OF COMBINATORICS 3 (1996), \#R24

Thus, for every green edge $p q, q \in M$ the vertex $q$ is incident to both red and blue edges. Let $a$ be an end vertex of a red-blue path $a b c \ldots$; we have $a \in M, b \in N$, $c \in M$. Say, the edge $b a$ is red, and $b c$ is blue. No green edge is incident to $a$. We perform one local replacement on $A$, replacing $b c$ by $b a$, and then delete the vertices $b$ and $a$. The system corresponding to the remaining graph still satisfies the property 2 , and has $n-1$ sets. Thus, by induction, the theorem is proved.

\section{Lower bounds on the distance}

We begin this section with a conjecture.

CONJECTURE. For every natural $n$ there exists $f(n)$ such that for every matroid $M$, if $\bar{x}=\left(x_{1}, \ldots, x_{n}\right)$ and $\bar{y}=\left(y_{1}, \ldots, y_{n}\right)$ are two locally equivalent transversals of a set system $\left(V_{1}, \ldots, V_{n}\right)$ in $M$ then the distance between $\bar{x}$ and $\bar{y}$ does not exceed $f(n)$.

I firmly believe this conjecture to be true. Trivially, $f(1)=1$. It is easy to prove (and is left to the reader as an exercise) that $f(2)=3$. The case $n=3$ can possibly be dealt with by a long and tedious but not very difficult argument.

On the other hand, the function $f(n)$ if it exists must grow at least exponentially. Below we shall construct examples proving this, and an example showing that $f(3) \geq$ 7 .

EXAMPLE 1. Let $M$ be a 3-dimensional space over any field; $a, b, x$ three linearly independent vectors. Set

$$
V_{1}=\{a, b\}, V_{2}=\{a, b, x\}, V_{3}=\{a+x, b+x, a+b\} .
$$

It is easy to check that this set system has eight independent transversals, and that the transversals $(a, b, a+x)$ and $(b, a, b+x)$ are at distance 7 .

EXAMPLE 2. For $i=1, \ldots, n$ let $V_{i}=\left\{e_{i}^{0}, e_{i}^{1}\right\}$ be $n$ disjoint sets of size 2; $M=\bigcup V_{i}$, the matroid structure on $M$ to be specified later.

The set $H=V_{1} \times \ldots \times V_{n}$ of $n$-tuples of elements of $M$ forms the Hamming graph; two $n$-tuples being adjacent whenever they differ in only one coordinate. Every matroid structure on $M$ determines a subgraph of $H$ induced on the vertices corresponding to independent subsets of $M$; and we need to choose a matroid structure on $M$ so that the diameter of some connected component of this graph be as big as possible. We shall use the following easy lemma.

LEMMA. For every set $V$, and every collection $\mathcal{X}$ of $k$-subsets of $V$ such that $\left|X_{1} \backslash X_{2}\right| \geq 2$ for any two different $X_{1}, X_{2} \in \mathcal{X}$ there exists a matroid on $V$ in which a $k$-set is independent if and only if it doesn't belong to $\mathcal{X}$.

PROOF. $\quad$ Let the bases of the matroid be the $k$-subsets of $V$ not belonging to $\mathcal{X}$. We only need to check that they satisfy the exchange axiom: 
For any two bases $X, Y$, and any $x \in X$ there exists $y \in Y$ such that $X \backslash\{x\} \cup\{y\}$ is also a base.

If $x \in Y$ then we can take $y=x$. If $X \backslash Y=\{x\}$ then $Y \backslash X=\{y\}$, and we replace $x$ by $y$. So, let $x \in X \backslash Y$, and $|Y \backslash X| \geq 2$, say, $\{y, z\} \subseteq Y \backslash X$. By our assumption on the collection $\mathcal{X}$ at least one of the sets $X \backslash\{x\} \cup\{y\}, X \backslash\{x\} \cup\{z\}$ does not belong to $\mathcal{X}$ and therefore is a base — the exchange property is proved.

We shall denote vertices of $H$ by $(0,1)$-vectors of length $n$; the vector $\left(\epsilon_{1}, \ldots, \epsilon_{n}\right)$ corresponding to the transversal $\left(e_{1}^{\epsilon_{1}}, \ldots, e_{n}^{\epsilon_{n}}\right)$. The condition on the collection $\mathcal{X}$ from the Lemma now means simply that $\mathcal{X}$ corresponds to an independent set of vertices of $H$.

Let $n$ be even, $n=2 m$. Denote by $H_{i}$ the set of vectors of weight $i$ : those having exactly $i$ coordinates equal to 1 .

We shall construct the set $\mathcal{X}=H_{m-2} \cup\left(H_{m} \backslash \mathcal{Y}\right) \cup H_{m+2}$ for some $\mathcal{Y} \subseteq H_{m}$ such that in the graph $H \backslash \mathcal{X}$ the set $\mathcal{Y}$ is contained in a connected compomnent of large diameter.

We define a graph on the set $H_{m}$; two vectors are adjacent if and only if they differ in exactly two coordinates. This is the Johnson graph $J(2 m, m)$. The vertices of any induced path of length $l$ in this graph form such set $\mathcal{Y}$ with the diameter of the connected component equal to $2 l$. So, we need to find long induced paths in the graphs $J(2 m, m)$. By a recent result of A.Evdokimov [2], one can find such paths of length $>(2-\epsilon)^{n}$ for any $\epsilon>0$ and large enough $n$. This proves that $f(n)$ grows faster than any exponent $(2-\epsilon)^{n}$.

The mentioned theorem of A.Evdokimov is new and yet unpublished but it is easy to prove an exponential (though worse) lower bound using a well-known result by the same A.Evdokimov [3] that in the binary Hamming graph of dimension $m$ one can find an induced path of length $c \cdot 2^{m}$. If $\left(v_{1}, \ldots, v_{l}\right)$ is such path then the sequence of vectors $\left(w_{1}, \ldots, w_{l}\right)$ where $w_{i}=v_{i} \bar{v}_{i}\left(\bar{v}_{i}\right.$ is the complement of $\left.v_{i}\right)$ gives an induced path in the graph $J(2 m, m)$ of the same length. Thus, $f(n) \geq c \cdot 2^{n / 2}$.

Finally, I conjecture that $f(n) \leq 2^{n}-1$. This conjecture is not supported by any evidence, and is much more dubious than the first one.

\section{References}

[1] A. Bouchet, Digraph decompositions and Eulerian systems. SIAM J. Alg. Disc. Meth. 1987, vol.8, no.3, 323-337.

[2] A. Evdokimov, private communication.

[3] A. Evdokimov, The maximal length of a chain in the unit $n$-dimensional cube. Mat. Zametki 1969, vol.6, 309-319. [Russian; English transl.: Math. Notes 1969, vol.6, 642-648] 
[4] D. Fon-Der-Flaass, Local complementations of simple and oriented graphs, Sibirskii Zhurnal Issledovanija Operacij 1994, vol.1, no.1, 43-62. [Russian; English transl. in: A.D.Korshunov (ed.), Discrete Analysis and Operations Research, Kluwer, 1996. 15-34] 